\title{
Flavonoids in Lemon and Grapefruit IntegroPectin**
}

\author{
Antonino Scurria, ${ }^{[\mathrm{a}]}$ Marzia Sciortino, ${ }^{[\mathrm{b}]}$ Lorenzo Albanese, ${ }^{[\mathrm{c}]}$ Domenico Nuzzo, ${ }^{[\mathrm{d}]}$ \\ Federica Zabini, ${ }^{[c]}$ Francesco Meneguzzo, ${ }^{[\mathrm{cc}]}$ Rosa Alduina, ${ }^{[\mathrm{e}]}$ Alessandro Presentato, ${ }^{[\mathrm{e}]}$ \\ Mario Pagliaro, ${ }^{*[a]}$ Giuseppe Avellone, ${ }^{*[b]}$ and Rosaria Ciriminna*[a] \\ Dedicated to Professor Zayniddin Muhidinov, National Academy of Sciences of Tajikistan.
}

\begin{abstract}
Following the analysis of terpenes present in new lemon and grapefruit "IntegroPectin" pectins obtained via the hydrodynamic cavitation of industrial lemon and grapefruit processing waste, the HPLC-MS analysis of flavonoid and other phenolic compounds reveals the presence of eriocitrin, naringin, hesperidin and kaempferol typical of the respective citrus fruits. The pectic fibers rich in rhamnogalacturonan-I regions act as chemical sponges adsorbing and concentrating at their outer
\end{abstract}

surface highly bioactive citrus flavonoids and terpenes. These findings, together with the unique molecular structure of these new whole citrus pectins, provide preliminary insight into the broad-scope biological activity of these new biomaterials. Numerous new biomedical applications are anticipated, including likely use in the prevention and treatment of microbial infections and neurodegenerative disease.

\section{Introduction}

Communicating the discovery of the high antioxidant activity and lack of cytotoxicity on human pulmonary cells of lemon pectin obtained via hydrodynamic cavitation $(\mathrm{HC})$ of citrus processing industrial waste, ${ }^{[1]}$ we named "IntegroPectin" this new citrus pectic biomaterial. Reporting shortly afterwards its antibacterial activity, largely superior to that of commercial

[a] Dr. A. Scurria, Dr. M. Pagliaro, Dr. R. Ciriminna

Istituto per lo Studio dei Materiali Nanostrutturati, CNR

via U. La Malfa 153

90146 Palermo (Italy)

E-mail: mario.pagliaro@cnr.it rosaria.ciriminna@cnr.it

[b] Dr. M. Sciortino, Prof. G. Avellone

Dipartimento di Scienze e Tecnologie Biologiche Chimiche e Farmaceutiche Università di Palermo

via Archirafi 32

90123 Palermo (Italy)

E-mail: beppe.avellone@unipa.it

[c] Dr. L. Albanese, Dr. F. Zabini, Dr. F. Meneguzzo

Istituto per la Bioeconomia, CNR

via Madonna del Piano 10

50019 Sesto Fiorentino (FI) (Italy)

[d] Dr. D. Nuzzo

Istituto per la Ricerca e l'innovazione Biomedica, CNR

via U. La Malfa 153

90146 Palermo, Italy

[e] Prof. R. Alduina, Dr. A. Presentato

Dipartimento di Scienze e Tecnologie Biologiche Chimiche e Farmaceutiche

Università di Palermo

viale delle Scienze, Ed.16

90128 Palermo (Italy)

${ }^{[*}$ A A previous version of this manuscript has been deposited on a preprint server ( $h$ ttps://doi.org/10.20944/preprints202102.0620.v1)

Supporting information for this article is available on the WWW under https://doi.org/10.1002/open.202100223

(c) 2021The Authors. Published by Wiley-VCH GmbH. This is an open access article under the terms of the Creative Commons Attribution Non-Commercial License, which permits use, distribution and reproduction in any medium, provided the original work is properly cited and is not used for commercial purposes. citrus pectin, we ascribed it to the "combined antibacterial action of lemon oil..., and of the water-soluble lemon flavonoids found in abundant amount at the surface of lyophilized IntegroPectin". ${ }^{[2]}$

Subsequent investigation of the terpenes adsorbed at the surface of these new whole lemon (and grapefruit) pectins, actually showed the presence of relatively high amount of highly bioactive $\alpha$-terpineol and terpinen-4-ol. ${ }^{[3]}$ We ascribed formation of the latter compounds during the HC-based extraction to limonene and linalool decomposition, catalyzed by residual citric acid in the citrus peel by-product of the industrial production of the juice. Further reporting the broadscope bactericidal activity of grapefruit IntegroPectin against Gram-positive and Gram-negative bacteria, and suggesting also the bacterial inactivation mechanism, ${ }^{[4]}$ we noted that the analysis of the flavonoids and terpenes present in the new IntegroPectin was necessary en route to develop new applications of these new pectic polymers. Indeed, along with the aforementioned pronounced antibacterial activity, ${ }_{1}^{[2,3]}$ grapefruit IntegroPectin was recently shown to exert high in vitro neuroprotective, mitoprotective and antiproliferative activity towards neuronal model cells. ${ }^{[5]}$ Lemon IntegroPectin, in its turn, shows exceptionally high in vitro neuro- and mitoprotective activity towards the same human neuron model cells. ${ }^{[6]}$

Having been obtained after hydrolysis with mineral acid in hot water followed by precipitation with isopropyl alcohol and by extensive washing, ${ }^{[7]}$ commercial citrus pectin does not contain either citrus terpenes or citrus flavonoids. Furthermore, the polymer structure of commercial pectin is extensively degraded, chiefly because of hydrolytic loss of the rhamnogalacturonan-I side chains ("hairy" structural region) bound to the main homogalacturonan chain of the heterogeneous polysaccharide. ${ }^{[8]}$

Flavonoids contained in large amount in Citrus fruits ${ }^{[9]}$ (with O-glycosylated flavonoids abundant in all fruit tissues and polymethoxylated flavonoids accumulating preferentially in the 
flavedo) ${ }_{1}^{[10]}$ and thus in citrus processing waste, are the target of specific extraction procedures. ${ }^{[11]}$ These biophenols are increasingly added to several new nutraceutical products and food supplements. It is now well known that their broad-scope biological activity, including anti-inflammatory, antiproliferation, anti-angiogenesis and cell cycle regulation effects, is due not only to their free radical scavenging activity, but also to their ability to act as modulators of several key molecular events implicated in cell survival and apoptosis. ${ }^{[12]}$

As recently emphasized by Valtchev and co-workers reviewing citrus flavonoid anti-cancer properties, ${ }^{[12]}$ it is also of relevance to this account the growing evidence of synergy between these bioactive molecules, with citrus peel whole extracts showing higher activity than the fractionated extracts or isolated single compounds. Finally, citrus peel extracts obtained from freeze-dried peel, due to enhanced rupture of the cell vacuoles and release of phenolic compounds accumulating therein, have $>50 \%$ higher concentrations of total phenolic and total flavonoid compounds when compared to extracts obtained from the fresh peel. ${ }^{[13]}$ Now, we report the qualitative and quantitative analysis of the main flavonoids and phenolic compounds accumulated at the surface of lemon and grapefruit IntegroPectin.

\section{Results and Discussion}

The IntegroPectin samples were obtained by freeze-drying the aqueous solution obtained after the HC-based extraction of lemon and grapefruit citrus processing waste (Scheme 1) using

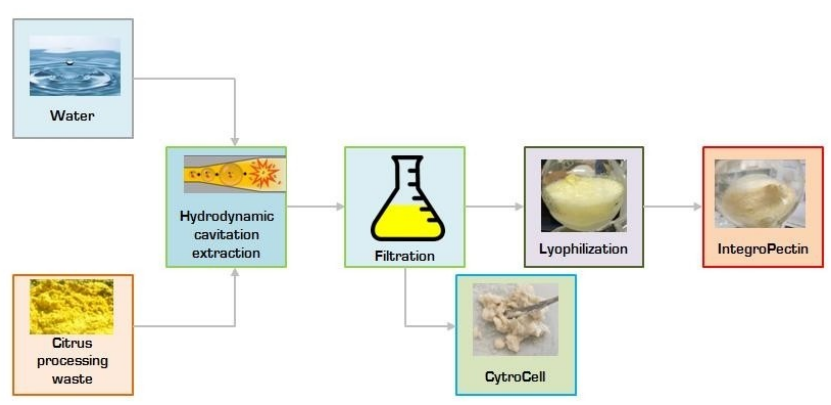

Scheme 1. The Citrus biorefinery scheme based on hydrodynamic cavitation.

\begin{tabular}{|lll|}
\hline $\begin{array}{l}\text { Table 1. Flavonoids in lemon and grapefruit IntegroPectin identified by } \\
\text { HPLC-MS. }\end{array}$ & $\begin{array}{l}\text { Lemon } \\
\text { IntegroPectin } \\
\text { Biophenol }\end{array}$ & $\begin{array}{l}\text { Grapefruit } \\
\text { IntegroPectin } \\
{[\mathrm{mg} / \mathrm{g}]}\end{array}$ \\
& - & 0.34 \\
\hline Naringenin & 0.11 & 73.66 \\
Naringin & 0.60 & 0.60 \\
Hesperidin & 3.35 & - \\
Eriocitrin & 0.26 & - \\
Kaempferol & 0.26 & 0.26 \\
Kaempferol-7-O-glucuronide & 0.28 & - \\
p-Coumaric acid & 0.56 & 0.31 \\
Gallic acid & & \\
\hline
\end{tabular}

water as the only solvent (for the soluble compounds) and dispersing medium (for the cellulosic insoluble fraction). ${ }^{[14]}$

Table 1 lists the flavonoid and other phenolic compounds found using the highly sensitive HPLC-MS technique employed (see the Experimental section). In agreement with the literature, the two main flavonoids found are eriocitrin (eriodictyol 7rutinoside) for lemon IntegroPectin, and naringin (4',5,7-trihydroxy flavanone 7-rhamnoglucoside) for grapefruit IntegroPectin. Particularly abundant in the lemon peel and also significantly present in the juice (but absent in the seed), eriocitrin is a powerful antioxidant with an activity level equal to that of $\alpha$ tocopherol. The latter activity is even enhanced in the presence of citric acid. ${ }^{[15]}$

Supported by clinical studies in pre-diabetic patients showing benefits in glycemic control, reduced systemic inflammation and oxidative stress, ${ }^{[16]}$ new nutraceutical products using lemon-derived eriocitrin as active ingredient for reducing blood glucose levels have been lately commercialized. ${ }^{[17]}$

Along with naringin, hesperidin (hesperetin 7-rutinoside) is also present in significant amount at the surface of lemon IntegroPectin. Administered with eriocitrin, hesperidin suppresses the oxidative stress in diabetic mice. ${ }^{[18]}$ Due to its broadspectrum bioactivity in the prevention of cancer, ${ }^{[19]}$ neurodegenerative, ${ }^{[20]}$ and cardiovascular ${ }^{[21]}$ diseases, this flavanone glycoside is the most studied citrus flavonoid in biochemistry and biomedical research.

Flavanone glycoside naringin (4',5,7-trihydroxyflavonone-7rhamnoglucoside), by far the predominant flavanone found in grapefruit, ${ }^{[22]}$ was found to be exceptionally concentrated in grapefruit IntegroPectin, approaching $74 \mathrm{mg} / \mathrm{g}$. For comparison, the highest yield values for naringin extracted from fresh or dry grapefruit albedo so far reported are $10.5 \mathrm{mg} / \mathrm{g}$ (for fresh albedo) and $43.28 \mathrm{mg} / \mathrm{g} .{ }^{[23]}$ Besides showing anti-cancer, cholesterol-lowering, anti-apoptotic, anti-atherogenic, anti-inflammatory and antioxidant activity, naringin is an antimicrobial agent inhibiting growth of bacteria such as Actinomyces naeslundii, Actinomyces viscosus, Aggregatibacter actinomycecomitans, Enterococcus faecalis, Escherichia coli, Staphylococcus aureus and Lactobacillus casei as well as of Candida albicans fungal species typical of the dental plaque. ${ }^{[24]}$ Remarkably, naringenin (the aglycone of naringin) was lately found by scholars in South Korea to exert powerful antimicrobial activity against methicillin-resistant Staphylococcus aureus and its mutant strain $\Delta$ agr. $^{[25]}$ The flavanone inhibits the microbial fatty acid synthesis, ${ }^{[25]}$ partly explaining the lately discovered bactericidal activity of grapefruit IntegroPectin against S. aureus. ${ }^{[4,2]}$

Kaempferol 7-O-glucuronide, namely kaempferol $\left(3,4^{\prime}, 5,7-\right.$ tetrahydroxyflavone) with a $\beta$-D-glucosiduronic acid residue attached at the 7-position, was found in similar amount $(0.26 \mathrm{mg} / \mathrm{g})$ in both citrus IntegroPectins studied. The latter is a tetrahydroxyflavone likely formed during cavitation via reaction of kaempferol in the presence of residual glucose and citric acid abundant in citrus processing waste, which was recently reported to have strong antimicrobial activity. ${ }^{[26]}$

Kaempferol, an health beneficial phytoestrogen, was found in lemon IntegroPectin only, in the same amount of its aforementioned O-glucuronide. Molecular mechanism investi- 
gations have shown that the flavanol modulates a number of intracellular signaling cascades that regulate cellular signal transduction pathways linked to apoptosis, angiogenesis, inflammation, metastasis, ${ }^{[27]}$ and allergic airway inflammation and associated asthma symptoms. ${ }^{[28]}$ The main problem limiting nutraceutical applications of kaempferol has been its poor bioavailability due to poor solubility in water. As argued for other citrus flavonoids, such as hesperidin, naringin and the respective aglycones, the $\mathrm{HC}$-based extraction and the concomitant complexation with pectic fibers in the lyophilized, highly soluble IntegroPectin biomaterial, ${ }^{[29]}$ could substantially relieve the limited bioavailability of citrus flavonoids remaining unabsorbed in the colon after oral administration, ${ }^{[30]}$ opening the way to effective nutraceutical applications of this new citrus pectic substance.

\section{Conclusion}

The analysis of the main flavonoids present at the surface of the newly obtained citrus pectins, namely lemon and grapefruit IntegroPectin, complements the analysis of the adsorbed volatile compounds unveiling the presence of highly bioactive flavonoids. ${ }^{[3]}$ Eriocitrin predominates in lemon IntegroPectin, followed by significant amounts of hesperidin, kaempferol, and kaempferol 7-O-glucuronide. In the case of grapefruit IntegroPectin, naringin is by far predominant, with minor amounts of hesperidin and naringenin.

Along with the unique molecular structure of these new pectins, with very low degree of esterification and high amount of RG-I "hairy" region, ${ }^{[6]}$ these results shed further light on the high bactericidal, antioxidant, antiproliferative and mitoprotective activity of grapefruit IntegroPectin ${ }^{[4,5]}$ and the neuro- and mitoprotective action of lemon IntegroPectin. ${ }^{[6]}$ In the former case, the IntegroPectin polymer chains rich in RG-I regions act as carriers of adsorbed naringin, $\alpha$-terpineol and linalool, ${ }^{[3]}$ which are powerful antibacterial agents particularly against Gram-positive bacteria, permeating the hydrophobic S. aureus cell membrane, ${ }^{[31]}$ with subsequent likely hydrolysis of naringin into highly antimicrobial naringenin. ${ }^{[22]}$

The neuro- and mitoprotective action of lemon IntegroPectin, in its turn, might therefore be due to the combined neuroprotective, antioxidant and anti-inflammatory action of eriocitrin in synergy with hesperidin and with $\alpha$-terpineol and terpinen-4-ol neuroprotective species abundant in the newly obtained citrus pectin..$^{[3]}$ Again, the physiological effects of these small biomolecules would act in combination with the RG-I enriched structure of lemon IntegroPectin polysaccharide favoring interaction with the cancer cells studied, ${ }^{[5]}$ including human pulmonary cells. ${ }^{[1]}$ The latter RG-I side-chains, indeed, play a central role in enhancing the anti-cancer, immunomodulatory, anti-hyperlipidemic, and anti-hyperglycemic activity. ${ }^{[32]} \mathrm{A}$ key physiological function of these pectin side-chains is their ability to inhibit the pro-metastatic protein galectin-3 by binding to its carbohydrate recognition domain. ${ }^{[33]}$

In light of forthcoming biomedical applications of these new pectic materials, it is also relevant that RG-I enriched citrus pectin forms gels both adding $\mathrm{Ca}^{2+}$ cations and under acid conditions via a gelation mechanism, different from that of commercial pectin, in which the neutral sugar side-chains improve network formation. ${ }^{[34]}$

In conclusion, due to its broad physiological activity, pectin has been recently called by Russia's scholars "an universal medicine" ${ }^{[35]}$ Numerous new biomedical applications of citrus IntegroPectin are anticipated, including likely use in the prevention and treatment of microbial infections and neurodegenerative disease. Preliminary evidence of synergy seems to emerge via the combined broad scope bioactivity of the i) citrus flavonoids identified in this study, ii) terpenes and other volatile compounds adsorbed at their surface,,$^{[3]}$ and the unique molecular structure of these new pectins isolated after freezedrying the cavitation-based aqueous extract. ${ }^{[4-6]}$ The results of further ongoing biological and structural investigations will be reported in due course.

\section{Experimental Section}

A $600 \mathrm{mg}$ sample of each (lemon and grapefruit) IntegroPectin obtained as described elsewhere, ${ }^{[1,3]}$ was weighted and added to a $100 \mathrm{~mL}$ flask. A $50 \mathrm{~mL}$ aliquot of an $\mathrm{EtOH} / \mathrm{H}_{2} \mathrm{O}$ mixture $(\mathrm{v} / \mathrm{v}, 4: 1)$ was added to each flask. The extraction was carried out under sonication $(150 \mathrm{~W})$ for $15 \mathrm{~min}$ using a Transsonic $460 \mathrm{H}$ ultrasonic bath (Elma Hans Schmidbauer, Singen, Germany) operating at $35 \mathrm{kHz}$ ultrasonic frequency. The bath temperature during sonication reached $40^{\circ} \mathrm{C}$. After sonication, the content of each flask was transferred in centrifuge tubes to undergo centrifugation at 10,000 rpm for $10 \mathrm{~min}$ in an Allegra X-22 R (Beckman Coulter, Brea, CA, USA) centrifuge, after which a small sample of the supernatant of both samples was added to glass vials for analysis.

The high performance liquid chromatography-mass spectrometry (HPLC-MS) analyses were conducted using a Alliance e2695 (Waters, Milford, MA, USA) HPLC system equipped with autosampler, degasser and column heater coupled with a Q-Tof Premier (Waters, Milford, MA, USA) quadrupole time of flight mass spectrometer. The compounds were separated by a Thermo Scientific (Thermo Fisher Scientific, Waltham, MA, USA) BetaBasic HPLC C18 column (50x $2.1 \mathrm{~mm}$ I.D., particle size $1.8 \mu \mathrm{m}$ ) kept at $20^{\circ} \mathrm{C}$ injecting each time a $5 \mu \mathrm{L}$ volume sample. All samples were injected in duplicate using a thermostated autosampler kept at $4{ }^{\circ} \mathrm{C}$. The HPLC analyses were carried out using a mixture of $0.1 \mathrm{wt} . \%$ aqueous formic acid and $0.1 \mathrm{wt} . \%$ formic acid methanolic solution at $0.25 \mathrm{~mL} / \mathrm{min}$ flow rate. Elution started with $95 \%$ aqueous formic acid and $5 \%$ methanol formic acid, isocratic for one minute. In the subsequent $14 \mathrm{~min}$ the solvent becomes $100 \% \mathrm{MeOH}$, remaining isocratic for the subsequent $5 \mathrm{~min}$ (from $\min 15$ to $\min 20$ ). After $30 \mathrm{~s}$, the eluting solvent mixture is reverted to $95 \%$ aqueous formic acid and $5 \%$ methanolic formic acid, and kept as such for another $30 \mathrm{~s}$. The whole run lasted $21 \mathrm{~min}$. Every sample was injected twice. The concentration values reported in Table 1 are the average of the two values measured.

Quantitation of quercetin, resveratrol, caffeic acid, gallic acid, eriocitrin, naringin, and hesperetin was carried out using commercial samples of the phenolic compounds obtained from Sigma Aldrich (Gallarate, Italy) as standards. For the remaining compounds, we used the calibration curve of quercetin for the detection of flavonoids, and that of resveratrol for the assessment of other biophenols. The MS experiments were performed on Q-Tof Premier using dynamic range enhancement (DRE) as acquisition mode that avoids MCP saturation keeping a fairly good sensitivity. 
This allows to correctly quantify very abundant as well as trace level compounds, providing results suitable for statistical analysis. Atmospheric pressure chemical ionisation (APCI) in negative mode was used under the following conditions: capillary, $2.0 \mathrm{kV}$; extraction cone, $2.0 \mathrm{~V}$; ion guide, $2.0 \mathrm{~V}$; source temperature $80^{\circ} \mathrm{C}$, cone gas, $\mathrm{N}_{2}$, flow $35 \mathrm{Lh}^{-1}$; desolvation gas, $\mathrm{N}_{2}$, flow $300 \mathrm{Lh}^{-1}$.

The following compounds were researched in both lemon and grapefruit IntegroPectin samples: quercetin, naringin, sinapinic acid, rutin, quercetin-3-glucuronide, naringenin, hesperidin, hesperetin, eriodictyol, eriocitrin, diosmin, caftaric acid, petunidin 3-O- $\left(6^{\prime \prime}-\right.$ acetyl)glucoside, cyanidin 3-O-glucoside, malvidin 3-O-pentoside, peonidin 3-O-hexoside isomer, resveratrol, resveratrol dimer, resveratrol trimer, resveratrol tetramer, resveratrol hexoside, kaempferol, kaempferol 7-O-glucuronide, quercetin-3-O-(6"-O-malonyl)-glucoside, quercetin-3'-O-glucuronide, kaempferol-3-O-glucoside, quercetin-3-O-galactoside, myricetin-3-O-hexoside, myricetin, epigallocatechin-3-O-coumarate, epicatechin-3-O-vanillate, epigallocatechin, procyanidin $\mathrm{B} 3$, catechin, procyanidin $\mathrm{B} 2$, procyanidin $\mathrm{C} 2$, isovanillic acid, gallocatechin, prodelphinidin dimer B type, catechin-7-Oglucoside, caffeic acid methyl ester, 3-caffeoyl-4-p-coumaroylquinic acid, ferulic acid, ellagic acid, $p$-coumaric acid, gallic acid ethyl ester, vanillic acid, caffeic acid, $p$-hydroxybenzoic acid, and gallic acid. Table S1 in the Supporting Information displays the molecular formula, retention time in the HPLC-MS experiments, and exact mass for the main flavonoids identified in lemon and grapefruit IntegroPectin. Figure S1 in the Supporting Information shows the mass chromatograms and exact mass of extracted ions for each tentatively identified compound (TIC) for flavonoids in lemon IntegroPectin.

\section{Acknowledgements}

We thank OPAC Campisi (Siracusa, Italy) for the generous gift of lemon and grapefruit processing waste from which the IntegroPectin materials were obtained.

\section{Conflict of Interest}

The authors declare no conflict of interest.

Keywords: citrus fruits · flavonoids · hydrodynamic cavitation IntegroPectin · pectins

[1] D. Nuzzo, L. Cristaldi, M. Sciortino, L. Albanese, A. Scurria, F. Zabini, C. Lino, M. Pagliaro, F. Meneguzzo, M. Di Carlo, R. Ciriminna, ChemistrySelect 2020, 5, 5066-5071.

[2] A. Presentato, A. Scurria, L. Albanese, C. Lino, M. Sciortino, M. Pagliaro, F. Zabini, F. Meneguzzo, R. Alduina, D. Nuzzo, R. Ciriminna, ChemistryOpen 2020, 9, 628-630.

[3] A. Scurria, M. Sciortino, A. Presentato, C. Lino, E. Piacenza, L. Albanese, F. Zabini, F. Meneguzzo, D. Nuzzo, M. Pagliaro, D. F. Chillura Martino, R. Alduina, G. Avellone, R. Ciriminna, Molecules 2021, 26, 51.

[4] A. Presentato, E. Piacenza, A. Scurria, L. Albanese, F. Zabini, F. Meneguzzo, D. Nuzzo, M. Pagliaro, D. Chillura Martino, R. Alduina, R. Ciriminna, Antibiotics 2020, 9, 586.

[5] D. Nuzzo, M. Scordino, A. Scurria, C. Giardina, F. Giordano, F. Meneguzzo, G. Mudò, M. Pagliaro, P. Picone, A. Attanzio, S. Raimondo, R. Ciriminna, V. Di Liberto, Int. J. Mol. Sci. 2021, 22, 9368
[6] D. Nuzzo, P. Picone, C. Giardina, M. Scordino, G. Mudò, M. Pagliaro, A. Scurria, F. Meneguzzo, L. M. Ilharco, A. Fidalgo, A. Presentato, R. Alduina, R. Ciriminna, V. Di Liberto, Antioxidants 2021, 10, 669.

[7] S. Y. Chan, W. S. Choo, D. J. Young, X. J. Loh, Carbohydr. Polym. 2017 $161,118-139$

[8] W. Wang, X. Wu, T. Chantapakul, D. Wang, S. Zhang, X. Ma, T. Ding, X. Ye, D. Liu, Food Res. Int. 2017, 102, 101-110.

[9] J. J. Peterson, G. R. Beecher, S. A. Bhagwat, J. T. Dwyer, S. E. Gebhardt, D. B. Haytowitz, J. M. Holden, J. Food Compos. Anal. 2006, 19, S74-S80.

[10] S. Wang, C. Yang, H. Tu, J. Zhou, X. Liu, Y. Cheng, J. Luo, X. Deng, H. Zhang, J. Xu, Sci. Rep. 2017, 7, 10549.

[11] K. Sharma, N. Mahato, Y. R. Lee, Rev. Chem. Eng. 2019, 35, 265-284.

[12] N. Koolaji, B. Shammugasamy, A. Schindeler, Q. Dong, F. Dehghani, P. Valtchev, Curr. Dev. Nutr. 2020, 4, nzaa025.

[13] N. F. I. Noor Azman, H. E. Khoo, M. R. Razman, Curr. Res. Nutr. Food Sci. 2019, 7, 331-339.

[14] A. Scurria, L. Albanese, M. Pagliaro, F. Zabini, F. Giordano, F. Meneguzzo, R. Ciriminna, Molecules 2021, 26, 596.

[15] Y. Miyake, K. Yamamoto, T. Osawa, Food Sci. Technol. Int. Tokyo 1997, 3 84-89.

[16] C. B. Ribeiro, F. M. Ramos, J. A. Manthey, T. B. Cesar, Phytother. Res. 2019, 33, 1921-1933.

[17] Ingredients by Nature gets blood sugar eriocitrin formulation patent, Nutraceutical Business Review, 13 February 2020. https://nutraceuticalbusinessreview.com/news/article_page/Ingredients_by_Nature_gets_blood_sugar_eriocitrin_formulation_patent/162344 (accessed September 29, 2021).

[18] Y. Miyake, K. Yamamoto, N. Tsujihara, T. Osawa, Lipids 1998, 33, 689695.

[19] V. Aggarwal, H. S. Tuli, F. Thakral, P. Singhal, D. Aggarwal, S. Srivastava, A. Pandey, K. Sak, M. Varol, A. Khan, G. Sethi, Exp. Biol. Med. 2020, 245, 486-497.

[20] M. Hajialyani, M. Hosein Farzaei, J. Echeverría, S. M. Nabavi, E. Uriarte, E. Sobarzo-Sánchez, Molecules 2019, 24, 648.

[21] L. Pla-Pagà, J. Companys, L. Calderón-Pérez, E. Llauradó, R. Solà, R. M. Valls, A. Pedret, Nutr. Rev. 2019, 77, 845-864.

[22] M. Bacanlı, A. Ahmet Başaran, N. Başaran, The Major Flavonoid of Grapefruit: Naringin, In Polyphenols: Prevention and Treatment of Human Disease, R. Ross Watson, V. R. Preedy, S. Zibadi (Ed.s), Academic Press, New York: 2018; pp. 37-44.

[23] M. M. Victor, J. M. David, M. C. K. Sakukuma, E. L. França, A. V. J. Nunes, Green Process. Synth. 2018, 7, 524-529.

[24] G. Gutiérrez-Venegas, J. A. Gómez-Mora, M. A. Meraz-Rodríguez, M. A. Flores-Sánchez, L. F. Ortiz-Miranda, Heliyon 2019, 5, e03013.

[25] H.-S. Song, S. K. Bhatia, R. Gurav, T.-R. Choi, H. Joong Kim, Y.-L. Park, Y.H. Han, J. Y. Park, S. M. Lee, S. L. Park, H. S. Lee, W. Kim, Y.-G. Kim, Y.-H. Yang, bioRxiv 2020, 03.08.983049.

[26] D. Singh, S. K. Sharma, R. Rani, S. Mishra, R. A. Sharma, Int. J. Pharm. Clin. 2011, 3, 30-34.

[27] A. Y. Chen, Y. C. Chen, Food Chem. 2013, 138, 2099-2107.

[28] M. Molitorisova, M. Sutovska, I. Kazimierova, J. Barborikova, M. Joskova, E. Novakova, S. Franova, Eur. J. Pharmacol. 2021, 891, 173698.

[29] F. Meneguzzo, R. Ciriminna, F. Zabini, M. Pagliaro, Processes 2020, 8, 549.

[30] M. Zhang, S. Zhu, W. Yang, Q. Huang, C.-T. Ho, Food Funct. 2021, 12, 3307-3323.

[31] F. Reifsteck, S. Wee, B. J. Wilkinson, J. Med. Microbiol. 1987, 24, 65-73.

[32] D. Wu, J. Zheng, G. Mao, W. Hu, X. Ye, R. J. Linhardt, S. Chen, Crit. Rev. Food Sci. Nutr. 2020, 60, 2938-2960.

[33] A. P. Gunning, R. J. Bongaerts, V. J. Morris, FASEB J. 2009, 23, 415-424.

[34] J. Zheng, J. Chen, H. Zhang, D. Wu, X. Ye, R. J. Linardt, S. Chen, Food Hydrocolloids 2020, 101, 105536.

[35] O. Zaitseva, A. Khudyakov, M. Sergushkina, O. Solomina, T. Polezhaeva, Fitoterapia 2020, 146, 104676.

Manuscript received: September 24, 2021

Revised manuscript received: September 29, 202 